\title{
Unusual presentation of psoriasis
}

\author{
Shaikhah Alanazi ${ }^{1}$, Nora Arafat ${ }^{2}$, Walid Alghamdi ${ }^{3}$
}

${ }^{1}$ Department of Dermatology, Riyadh, Saudi Arabia, ${ }^{2}$ Department of Family Medicine, Security Forces Hospital, Riyadh, Saudi Arabia, ${ }^{3}$ Department of Dermatology, Security Forces Hospital, Riyadh, Saudi Arabia

Corresponding author: Dr. Shaikhah Alanazi, E-mail: shikahmd@hotmail.com

\begin{abstract}
Psoriasis is a widely prevalent disease, a chronic disorder with genetic predisposition and many environmental triggering factors. It has various clinical presentations. Typical lesions are recurring, chronic, scaly erythematous papules and plaques. Clinical presentation differs among patients, from those with only few localized plaques over extensors to those with generalized skin involvement. We report a 28 -year-old man who presented with unusual clinical presentation of psoriasis.
\end{abstract}

Key words: Psoriasis; Unusual presentation; Whorled presentation

\section{INTRODUCTION}

Psoriasis is an autoimmune polygenic skin disorder. Many triggering factors, e.g. infection, trauma or drugs, may elicit the disease in susceptible individuals [1].

The classic lesion is a well demarcated erythematous scaly plaque; these plaques can be limited or widespread in distribution. It is a dynamic disease; associated morphological changes and the development of a new lesion into an advanced plaque can slowly enlarge or remain static [2].

Here we discuss an unusual presentation of psoriasis that was managed as inflammatory linear verrucous epidermal nevus for years before reaching the diagnosis.

\section{Case Presentation}

A 28 -year-old Saudi male, without notable medical history, presented in 2008 with a chronic itchy scaly cutaneous lesion involving the trunk in whorled pattern for eight years. The lesion was stable with no changes for several years, not associated with joint pain or other systemic symptoms. Patient has a positive family history of psoriasis. He refused to have a skin biopsy at that time and diagnosed as inflammatory linear verrucous epidermal nevus
(ILVEN)based on the clinical presentation, with no scalp or nail changes.

In 2013, patient presented again to dermatology clinic with new skin lesions involving extremities. Skin punch biopsy taken from the left side of the trunk and labs were done. Biopsy result showed mild acanthosis with epidermal spongiosis, hyperkeratosis with parakeratosis, elongated rete ridges and neutrophilic infiltrate in stratum corneum. In the dermis, the capillaries are elongated and tortuous, extending upward into dermal papillae. A minimal perivascular infiltrate is seen that consist primarily of lymphocytes. All Labs results were within normal range.

Patient managed as case of psoriasis, started on medium potent topical corticosteroid over active lesions (mometasone furoate ointment, $1 \%$ twice daily)and topical calcipotriol (Diavonex cream twice daily). Narrow-band ultraviolet B light was offered and discussed with the patient but he refused.

Patient missed the follow up and presented again in 2016 reporting new skin lesions over the lower limbs.

By examination there are multiple erythematous whorled and linear scaly plaques involving both trunk and limbs respectively (Figs. 1 and 2). Scalp has 


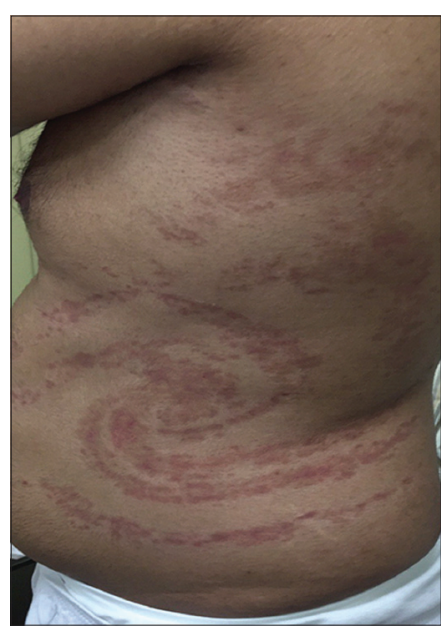

Figure 1: Note the whorled pattern of psoriatic plaques.

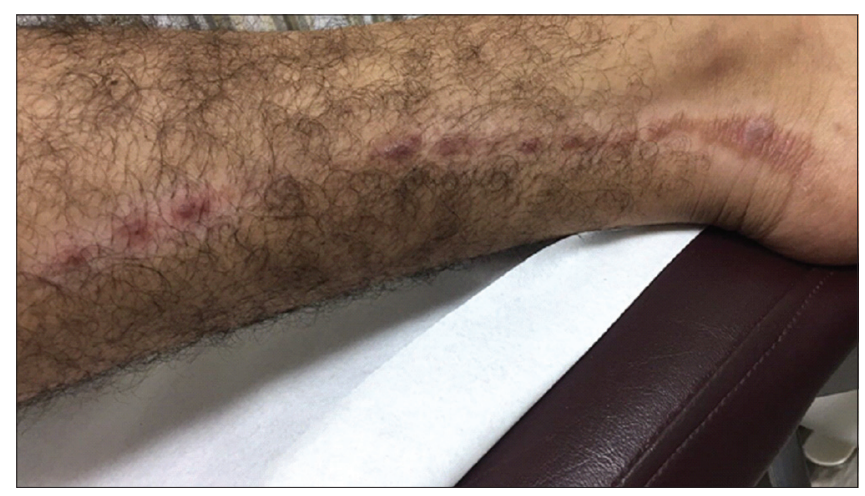

Figure 2: Linear arrangement of psoriatic papules and plaques that mimic ILVEN.

multiple yellowish well adherent scaly plaques over erythematous base mainly involving hair line. Nails have multiple deep irregular pitting and no Joints involvement.

Started on narrow-band ultraviolet B light three times \week plus the topical medications.

Now he is following up in the dermatology clinic regularly with good subjective and objective improvement.

\section{DISCUSSION}

Psoriasis is a chronic inflammatory disease characterized by altered immune responses, with dysregulated production of proinflammatory and anti-inflammatory cytokines $[3,4]$.

Typically has a chronic, persistent course with a reported incidence of 2-3\% worldwide [5]. It can occur at any age; majority of cases occur before 35 years, $10-15 \%$ of new cases begin in children younger than 10 years. Psoriasis is characterized by well-defined scaly, erythematous cutaneous plaques that vary in extent from few patches to generalized involvement. Psoriasis impact on patient's life is significant [6].

The International Psoriasis Council identifies four main types of psoriasis: plaque-type, guttate, generalized pustular Psoriasis, and erythroderma. Other several subphenotypes according to distribution (localized vs. widespread), onset (early vs. late), size (large vs. small) and thickness (thick vs. thin) of plaques, anatomical localization (flexural, scalp, palms/soles/nail), and disease activity (active vs. stable) [2].

Plaque psoriasis is the most common form (around 90\% of patient with psoriasis). Characterized by erythematous, well defined plaques with silvery micaceous scales. Plaques generally located on the extensor surface of knees and elbows, scalp, and in the lower back, but can affect any area of the body.

Guttate psoriasis is characterized by multiple to numerous scaly papules and plaques involving mainly the trunk and upper limbs. It has a sudden onset, within 2-4 weeks after a bacterial upper respiratory tract infection, mostly beat hemolytic streptococcal pharyngitis in pediatrics and adolescence age groups [2]

Generalized Pustular Psoriasis is a relatively rare but can be life-threatening disease. Characterized by widespread cutaneous involvement in form of pustules admixed with the psoriatic plaques and systemic manifestation like fever, lethargy, and neutrophils leukocytosis. Acute attacks can be triggered by infection, pregnancy, hypocalcemia, exposure to or withdrawal of medication.

Erythrodermic psoriasis represents one of the rarest form and it carries substantial morbidity and mortality [7].

Characteristic nail findings occur in 10-80\% of psoriatic patients and are more common in people with psoriatic arthritis [8].

Typical histologic findings include hyper- and parakeratosis, acanthosis with regular elongated rete ridges, hypogranulosis, dilated blood vessels in papillary dermis and a perivascular infiltrate of lymphocytes with neutrophils singly or within aggregates in the epidermis [6]. 
www.odermatol.com

\section{REFERENCES}

1. Nestlé FO, Kaplan DH, Barker J. Psoriasis. N Engl J Med. 2009;361:496-509.

2. Griffiths CE, Christophers E, Barker JN, Chalmers RJ, Chimenti S, Krueger GG, et al. A classification of psoriasis vulgaris according to phenotype. Br J Dermatol. 2007;156:258-62.

3. Lowes MA, Bowcock AM, Krueger JG. Pathogenesis and therapy of psoriasis. Nature. 2007;445:866-73.

4. Coimbra S, Figueiredo A, Castro E, Rocha-Pereira P, Santos-Silva A. The roles of cells and cytokines in the pathogenesis of psoriasis. Int J Dermatol. 2012;51:389-98.

5. Naldi L, Williams HC. Strachan Ded Inflammatory skin diseases IV: psoriasis The Challenge of Dermato-Epidemiology. Boca Raton, Fla CRC Press1997; 175- 190.
6. Christophers E. Psoriasis-epidemiologyandclinical spectrum. Clin Exp Dermatol. 2001;26:314-20.

7. Boyd AS, Menter A. Erythrodermic psoriasis. Precipitating factors, course, and prognosis in 50 patients. J Am Acad Dermatol. 1989;21:985-91.

8. Robert C, Kupper TS. Inflammatory skin diseases, T cells, and immune surveillance N Engl J Med. 1999;3411817-28.

Copyright by Shaikhah Alanazi, et al. This is an open-access article distributed under the terms of the Creative Commons Attribution License, which permits unrestricted use, distribution, and reproduction in any medium, provided the original author and source are credited.

Source of Support: Nil, Conflict of Interest: None declared. 